\title{
Influence of crystalline orientation on the thermal-energy convertion efficiency in shape memory alloys
}

\author{
J. Cortés-Pérez ${ }^{1, a}$, J.J.A. Ríos-Cortés ${ }^{1}$, F.M. Sánchez A², F.N. García $C^{1}$ and A Reyes $S^{3}$ \\ ${ }^{1}$ Laboratorio de Mecánica Aplicada, Centro Tecnológico Aragón, FES Aragón, Universidad Nacional \\ Autónoma de México, Av. Rancho Seco s/n, Col. Impulsora, Cd. Nezahualcoyotl, Edo. de México, \\ México \\ ${ }^{2}$ Instituto de Investigaciones en Materiales, Universidad Nacional Autónoma de México, Cd. \\ Universitaria, México \\ ${ }^{3}$ Centro de Investigación Multidiciplinaria Aragón, FES Aragón, Universidad Nacional Autónoma de \\ México, Av. Rancho Seco s/n, Col. Impulsora, Cd. Nezahualcoyotl, Edo. de México, México
}

\begin{abstract}
A theoretical study about the influence of crystalline orientation on the energy conversion efficiency of two shape memory alloys is present. The study consider single crystal samples in simple tension. The results showed higher properties for of the NiTi than $\mathrm{Cu}-\mathrm{Al}-\mathrm{Be}$, especially in the temperature-increment $(\Delta \mathrm{T})$ necessary for get a conversion energy cycle. However, the study showed a crystalline orientation favorable for $\mathrm{Cu}-\mathrm{Al}-\mathrm{Be}$.
\end{abstract}

\section{Introduction}

As it is well known an important application of shape memory materials is the designing of devices capable to transform thermal energy in to mechanical energy at low temperatures [1]. Also as it is known, the above mention, cover a great range of cases as the recover energy in conventional thermal machines and the conversion of solar energy in very single way [2]. Some authors has developed mathematical model for evaluate the energy conversion efficiency. Basically the models consider a fix and a removable loads, their strains associated and the work cycle in the stress-strain diagram [3]. These models have been capable to determinate efficiency parameters for springs, wires and sheet made with shape memory alloys [1-3]. Nevertheless nobody considers crystalline orientation and their possible influence in the energy conversion efficiency. In this work an analysis of the influence of the crystalline orientation in the energy conversion efficiency in two $\mathrm{SMA}$; $\mathrm{Cu}-\mathrm{Al}-\mathrm{Be}$ and $\mathrm{Ni}-\mathrm{Ti}$ is present.

\section{Develop}

For our analysis we consider single crystals samples in simple tension case which is easy represented in stress-strain diagrams as is shows in the Fig. 1. In this figure have been plotted a energy conversion

\footnotetext{
${ }^{\text {a }}$ Corresponding author: jacop@unam.mx
}

This is an Open Access article distributed under the terms of the Creative Commons Attribution License 4.0, which permits unrestricted use, distribution, and reproduction in any medium, provided the original work is properly cited. 


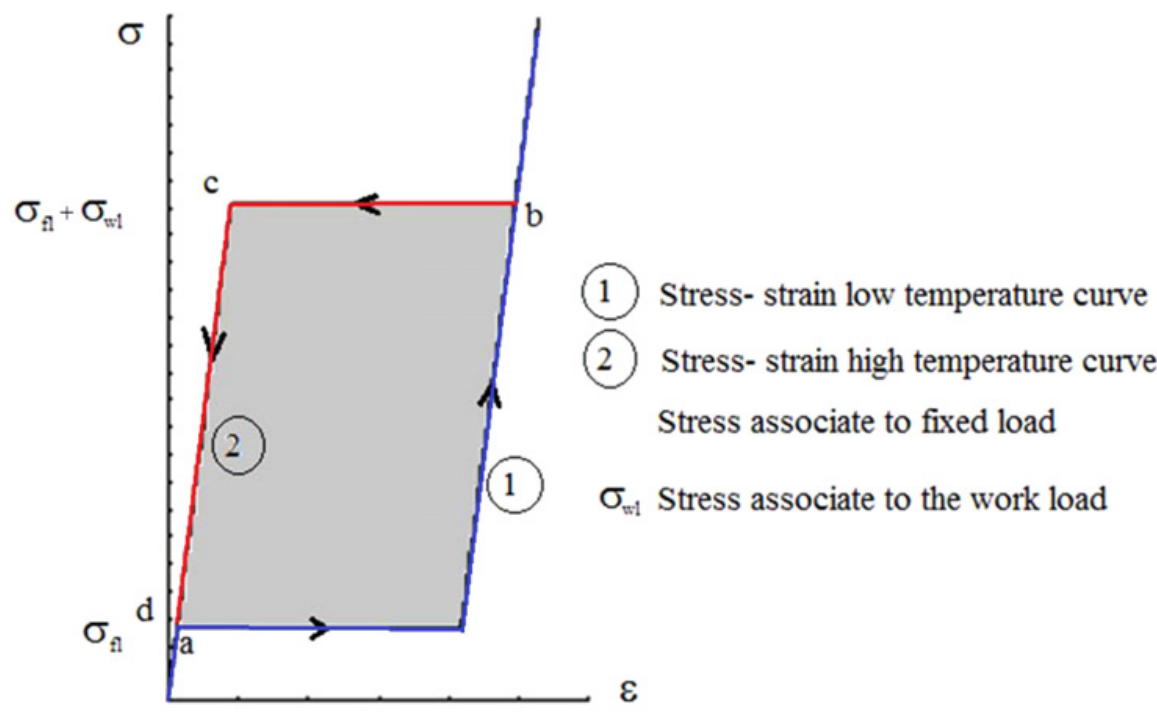

Figure 1. Energy conversion cycle in a stress-strain diagram.

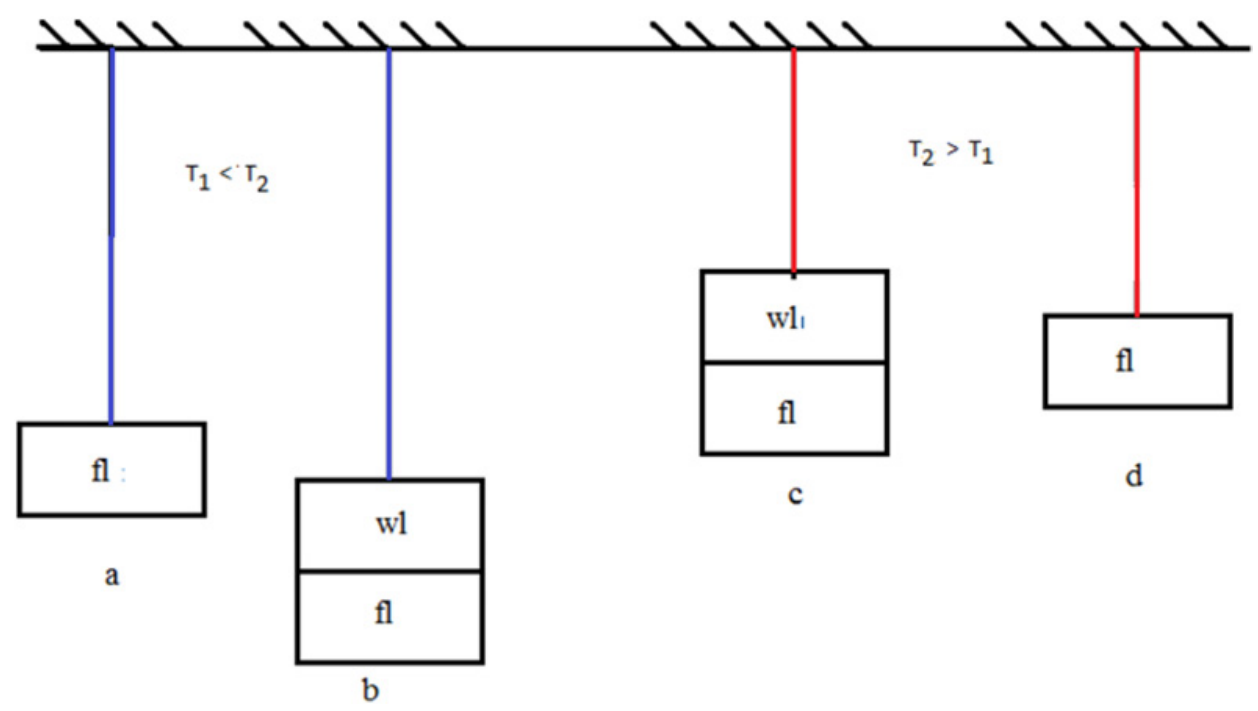

Figure 2. Energy conversion cycle in a schematic diagram associate to Fig. 1.

cycle with two curves that correspond to: a low and a high temperature cases. As is clear in Fig. 1 the parameters considered were: stress associate to fixed and work loads and low and high temperatures, both above to Af. Figure 2 shows a schematic diagram of the energy conversion cycle related to the states of the Fig. 1. Other parameters consider in the analysis are those properties of own alloys: Stress ratio, transformational system and transformation temperatures.

Our study consisted in the calculus of the specific energy obtained in a cycle for two shape memory alloys: $\mathrm{Ni}-\mathrm{Ti}$ and $\mathrm{Cu}-\mathrm{Al}-\mathrm{Be}$ with several crystalline orientation around to main direction in 


\section{$3^{\text {rd }}$ European Energy Conference}

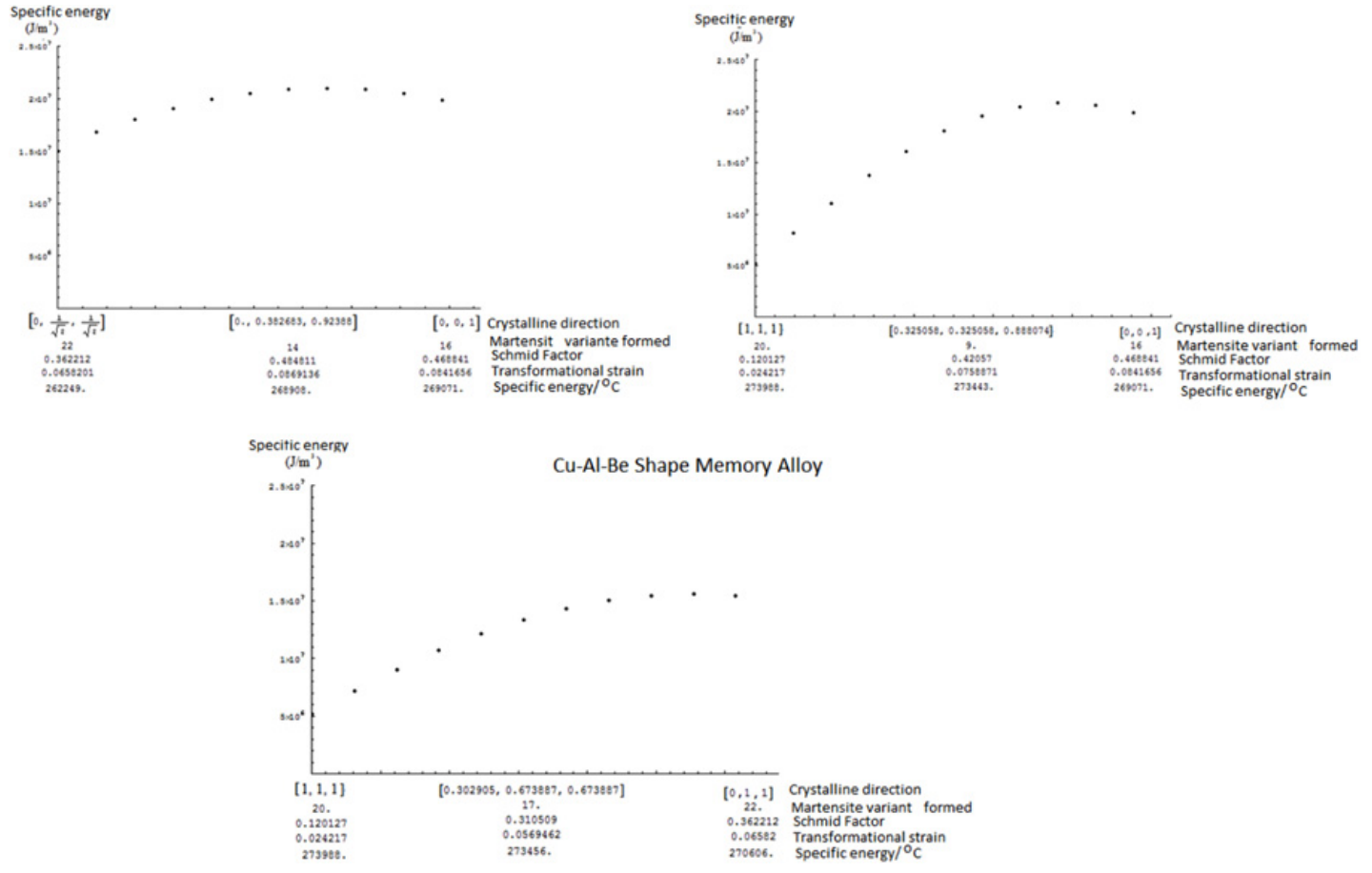

Figure 3. Specific energy converted for $\mathrm{Cu}-\mathrm{Al}-\mathrm{Be}$ SMA.

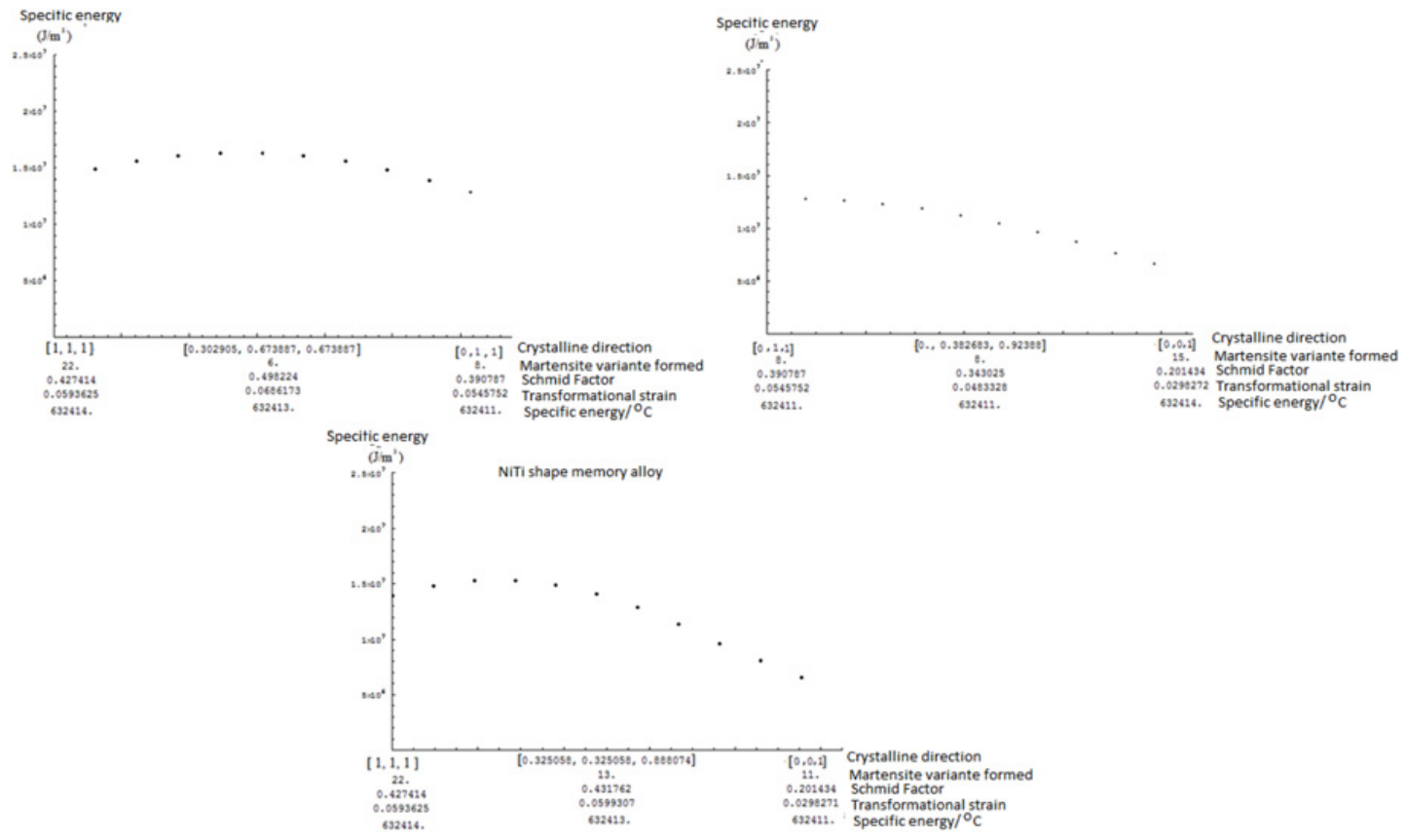

Figure 4. Specific Energy converted for Ni-Ti SMA. 


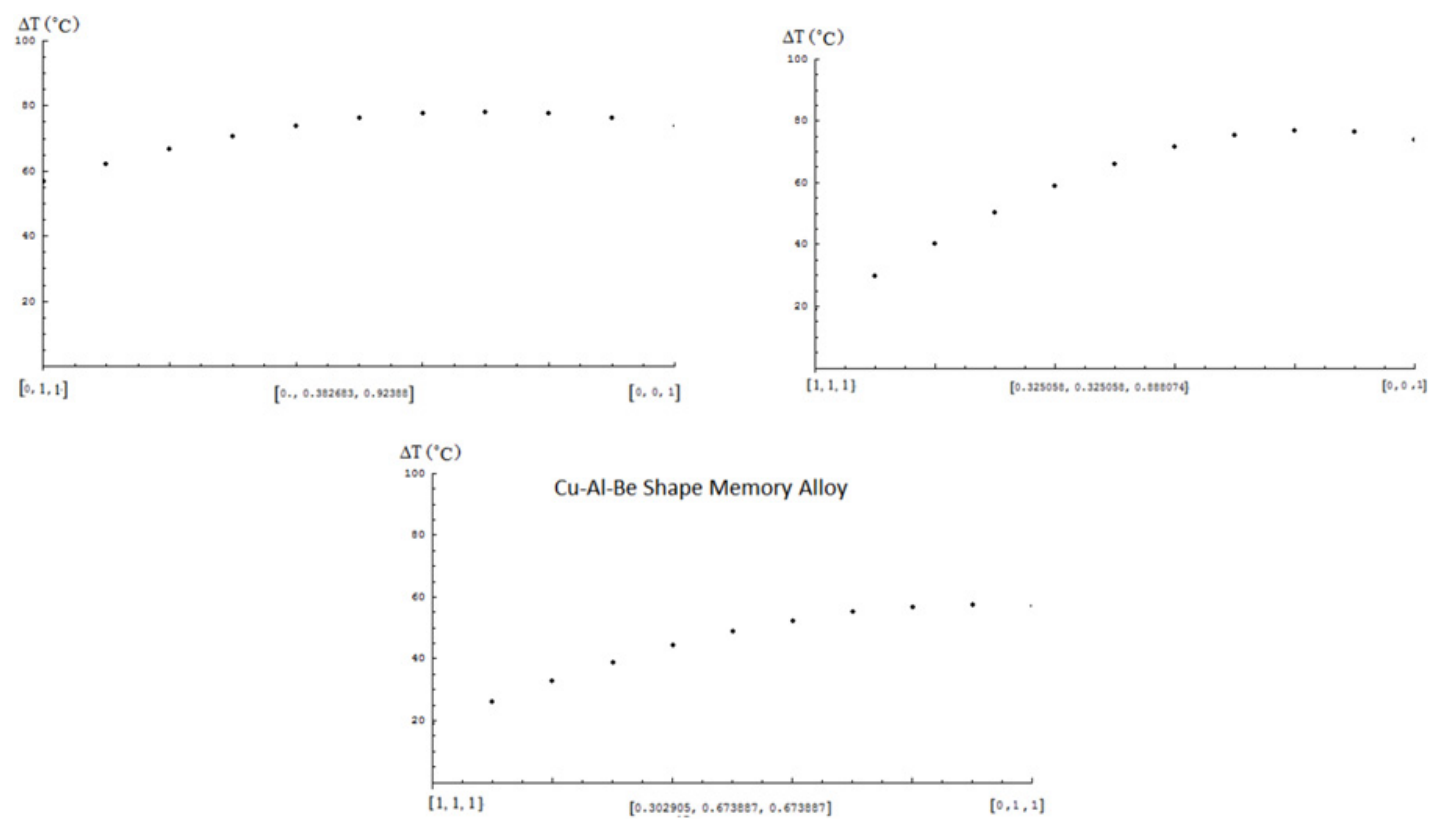

Figure 5. Temperature increse necesary for a Energy conversion cycle for $\mathrm{Cu}-\mathrm{Al}-\mathrm{Be} \mathrm{SMA}$.

the stereographic diagram of the austenitic phase of the both alloys; from [ $\left[\begin{array}{lll}0 & 0 & 1\end{array}\right]$ to $\left[\begin{array}{lll}0 & 1 & 1\end{array}\right]$, from $\left[\begin{array}{ll}0 & 0\end{array}\right.$ $\left.\begin{array}{ll}0 & 1\end{array}\right]$ to $\left[\begin{array}{lll}1 & 1 & 1\end{array}\right]$ and from [ $\left[\begin{array}{lll}0 & 1 & 1\end{array}\right]$ to $\left[\begin{array}{lll}1 & 1 & 1\end{array}\right]$. The calculus were made employing a mathematical model developed previously by one author of this job [4].

\section{Results}

For certain parameters proposed here, the Figs. 3 and 4 shows specific energy variation with the crystalline orientation variation for 3 paths along the main orientations of stereographic projection of austenitic phase structure.

As it was expected Ni-Ti SMA presented a higher energy by volume unit than the $\mathrm{Cu}-\mathrm{Al}-\mathrm{Be}$ SMA for any one crystalline orientation. In fact the energy by volume unit correspond to a cycle of energy conversion for $\mathrm{Ni}-\mathrm{Ti}$ is twice than for $\mathrm{Cu}-\mathrm{Al}-\mathrm{Be} ; 600000(\mathrm{~J} / \mathrm{m} 3)$ and $300000(\mathrm{~J} / \mathrm{m} 3)$ respectively. However it is not the principal advantage of NiTi SMA.

As is shows in the Figs. 5 and 6 the temperature-increment necessary to reach the work cycle for any one crystalline orientation is around $15^{\circ} \mathrm{C}$ for Ti-Ni while for $\mathrm{Cu}-\mathrm{Al}$-Be for most crystalline-direction is around $60^{\circ} \mathrm{C}$ except for crystalline direction near to [111] which the temperature increment for reach the work cycle is $20^{\circ} \mathrm{C}$.

This result never have been reported before however it is well know that the SMA's are high anisotropic. Their importance is due to the energy conversion for a SMA with high temperatureincrement will be very difficult if his mass is great. The last is a hard restriction for designing of devices which convert thermal energy into mechanical energy employing SMA. 
$3^{\text {rd }}$ European Energy Conference
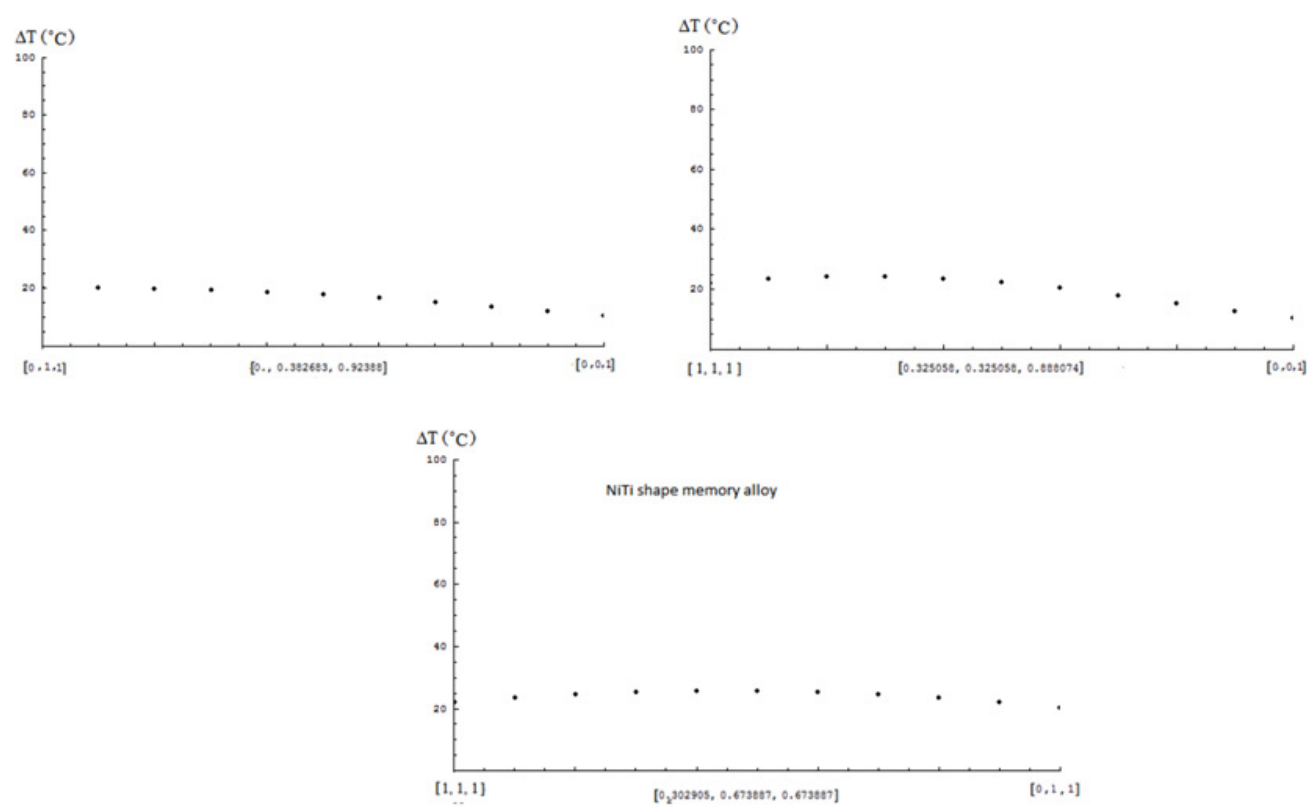

Figure 6. Temperature increase necessary for a Energy conversion cycle for $\mathrm{Cu}-\mathrm{Al}-\mathrm{Be} \mathrm{SMA}$.

\section{Conclusions}

The results obtained here shows that NiTi is more efficient to convert energy in a simple tension cycle than $\mathrm{Cu}-\mathrm{Al}-\mathrm{Be}$ because:

- The specific energy is twice.

- The temperature increase necessary for a energy conversion cycle is around $15^{\circ} \mathrm{C}$ for any crystalline direction while for the second is about $60^{\circ} \mathrm{C}$ for almost crystalline direction except to these near to $\left[\begin{array}{lll}1 & 1 & 1\end{array}\right]$.

The authors wish to thanks the financial support to PAPIIT-UNAM number IT102414 and the technical help of: Eng. Alberto Higuera García and C. Antonio González Montaño.

\section{References}

[1] Hisaaki Tobushi, Kousuke Date, Kouji Miyamoto. Journal of Solid Mechanics and Materials and Engineering. 4,.7. pp 1094. (2010)

[2] Alan L. Browne, Paul W. Alexander, Nilesh Mankame, Patrick Usoro, Nancy L. Johnson, Jan Aase, Peter Sarosi, Andrew C. Keefe Geoffrey P. Mcknight, Guillermo Herrera, Christopher Churchill,d, John Shaw, Jeffrey Brown..International workshop smart materials, structures \& ndt in aerospace Conference 2-4 de noviembre del 2011, Montreal, Quebec, Canada. (2011)

[3] J. J. Zhu, N. G. Liang, K. M. Liew and W. M. Huang. Journal of Intelligent Material Systems and Structures 12.pp.127. (2001)

[4] J. Cortés-Pérez. PhD Thesis PMyDI- UNAM. (2007) 